\title{
AUTOMATIC POSITION CONTROL OF THE SOLAR CELL
}

\author{
L. Magnuszewski*, K. Sapiołko**, M. Rećko ${ }^{* * *}$
}

\begin{abstract}
Automatic position control of the solar cell (APCoSC) is a project of the system, whose task is to direct the surface of the photovoltaic cell perpendicular to the sunrays falling on it and to follow the sun. The main parts are the base and pole, on which the cell is mounted. The base consists of a fixed part that provides a stable mounting on the ground, and a rotating part with mounted motor, which can perform a full $360 *$ rotation. The mast ends with a handle which, can tilt the solar panel automatically up to 90 degrees vertically by using a motor. The sun position sensor is built of 4 ambient light photosensors (APDS) arranged in the shape of a plus, enclosed in a casing, to which the sun's rays fall through the round opening. The case can be attached directly to the solar cell, or a handle using an adapter, in such a way as to form a parallel plane with the cell. The STM32 microcontroller manages the system and engine operation. The sun position sensor can work at a temperature of -30 to 85 Celsius, which ensures its universal use in many places on the earth. The photovoltaic cell produces the most energy when the sun's rays fall perpendicular to its surface, which is why the automatic tilt adjustment is used. In large cities, APCoSC is used on flat roofs of houses, where there are many obstacles such as other buildings around. The system adapts itself to the position of the sun and selects the most favourable position of the solar panel in real time.
\end{abstract}

Keywords: photovoltaic cells, sun sensor, position control.

\section{Introduction}

The use of fossil fuels for the production of electricity is associated with significant $\mathrm{CO} 2$ emissions to the atmosphere. It contributes to global warming and environmental pollution. In order to save the environment, renewable energy sources such as wind or photovoltaic panels have been used (Rocio et al., 2018, J. Kern M. Russell 1978). Accelerated growth in electricity demand while reducing the use of fossil fuels has forced the use of renewable energy sources. More and more farm solar panels are being built. However, photovoltaic panels are permanently mounted, which means that they do not use full power for the day cycle and thus their efficiency is low. To use the full power, it is necessary to set the panel plane perpendicular to the incidence of sunlight. For this purpose, two-axis bases controlled by a microcontroller are used (Allamehzadeh 2016, Deb and Roy 2012). The solutions used on the market, however, are expensive and significant in their dimensions. Therefore, the goal of our project is to design a solar tracking system that is simple and cheap.

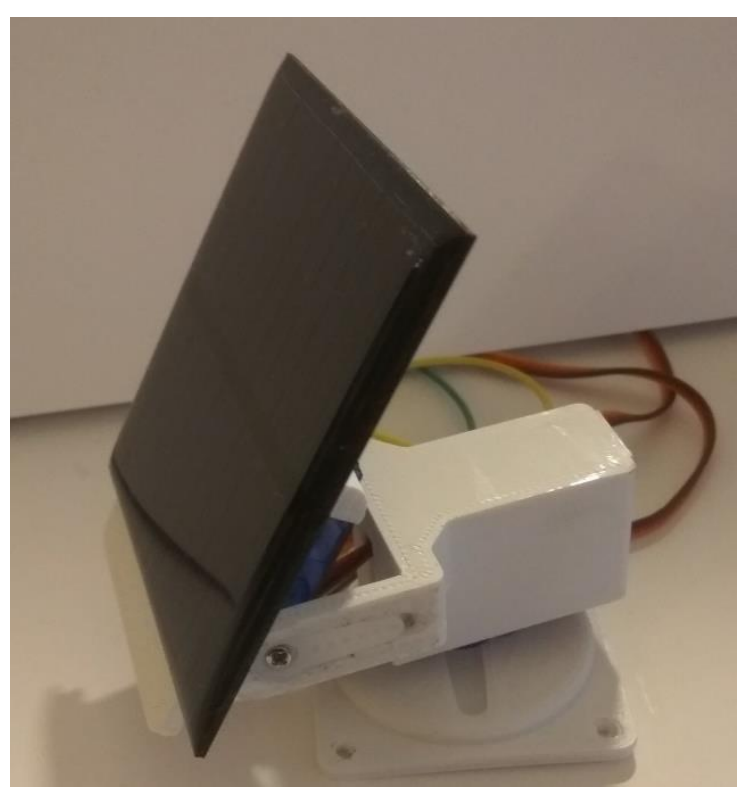

Fig. 1: Solar tracker - test platform This system would be applied to flat roofs of houses or administrative buildings.

\footnotetext{
Łukasz Magnuszewski Ołdakowski, Bialystok University of Technology, Wieska 45C; 15-351, Bialystok; PL, lukasz.magnuszewski123@gmail.com

** Karol Sapiołko, Bialystok University of Technology, Wiejska 45C, 15-351, Bialystok, PL, karolsa20@wp.pl

*** Maciej Rećko, MSc Eng.: Bialystok University of Technology, Wiejska 45C, 15-351 Bialystok, PL, m.recko@ outlook.com
} 


\section{Materials and research methods}

The test platform is shown in Figure 1. The platform can automatically rotate and automatically adjust the inclination angle of the solar panel. It was printed using the 3D method using PVC. We used two servo motors and four APDS sensors. These elements are connected to the STM32 F767ZI microcontroller. The solar panel has been connected to a digital voltage meter.

\section{Mechanics}

Figure 1. shows The Solar Tracker test model, designed in the SolidWorks program. It is made of a photovoltaic panel, a revolving base with a mounted servomotor, a movable solar panel holder with a mounted servomotor. Solar Tracker has two axes of freedom, left-right and up-down (Ozcelik et al., 2011). Four sensors mounted in the upper part are embedded in four quadrants of the circle, separated from each other by cross-shaped walls

\section{Equipment}

Tab. 1: Mini photovoltaic panel specification

The STM32 board is connected to the APDS sensors by means of the $\mathrm{I} 2 \mathrm{C}$ bus. It reads the intensity of light that falls on them, and then PWM signals are sent to the contacts of servomotors with a specific pulse width depending on the measured light intensity. A digital voltage meter is connected to the PV panel.

\section{Solar panel}

Table 1 presents its specification. The reason for choosing this model of a photovoltaic panel was its low price and small dimensions. Thanks to this, the test platform is easy to transport.

\section{Sensors}

Figure 2 shows the location of the sensors. The task of four APDS sensors separated from each other is the location of the sun's position. The selected sensors are cheap and reliable. The algorithm once detected lower light intensity at one of the sensors, automatically corrects the setup of the device. The sensors are connected via the I2C bus to the STM 32 board. They send a value from 0 to 65536 because the encoding is 16 bit. Its specification is presented in table 2 .

\section{Servomotors}

The servo motors (MG90) used in the test platform are used to

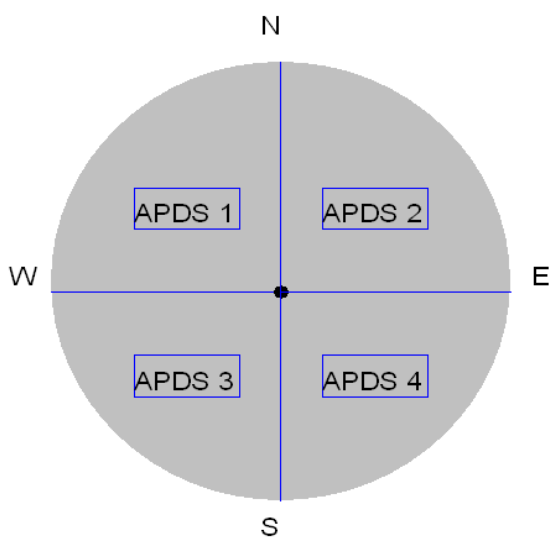

Fig. 2: Location of the sensors adjust the platform's inclination and rotation. The servomotor mounted in the base has the possibility of rotation from east to west $\left(0-180^{\circ}\right)$, and the servomotor mounted in the handle of the solar panel controls the inclination angle $\left(0-180^{\circ}\right)$. The servomotors are connected to the STM32 board using 3 wires $(+5 \mathrm{~V}$, GND, PWM). There was no need to use an engine controller.

\section{STM32}

In this project, we used the STM32 F767ZI microcontroller. However, any model of this microcontroller would be perfect for implementing the Solar Tracker. The selected model supports 4 I2C buses and 10 pins to generate the PWM signal. It is also possible to develop this project by using an Ethernet port for communication of the STM32 board and the computer. It is then possible to read the voltage after using the voltage divider. 


\section{Program}

The program that was written to track the position of the sun works in two stages. The first stage is reading the light intensity value from 4 APDS sensors. Then these values are compared with each other, for example, the values of sensors up down. If a difference exceeds set value is detected (set to eliminate noise and interference), a PWM signal is generated with a defined pulse width that controls the servomotor to take a step left (up) or right (down). The pulse width is calculated based on the preceding pulse width operation and is increased or decreased.

The second stage of the program is data archiving every 30 minutes. We archive the intensity of sunlight, the width of the PWM fill, and the time with the help of the Real Time Clock built into STM32. Thanks to this application, we introduced the correction of the Solar Tracker position. When the sun sets down, the sensors do not provide the control signals. The Solar Tracker returns to the first archived setup to enable the quickest illumination of tracking sensors as soon as the sun rises. The data can be archived in STM32 RAM, which will cause them to be overwritten each subsequent day, or on a microSD card (Makhija et al., 2017).

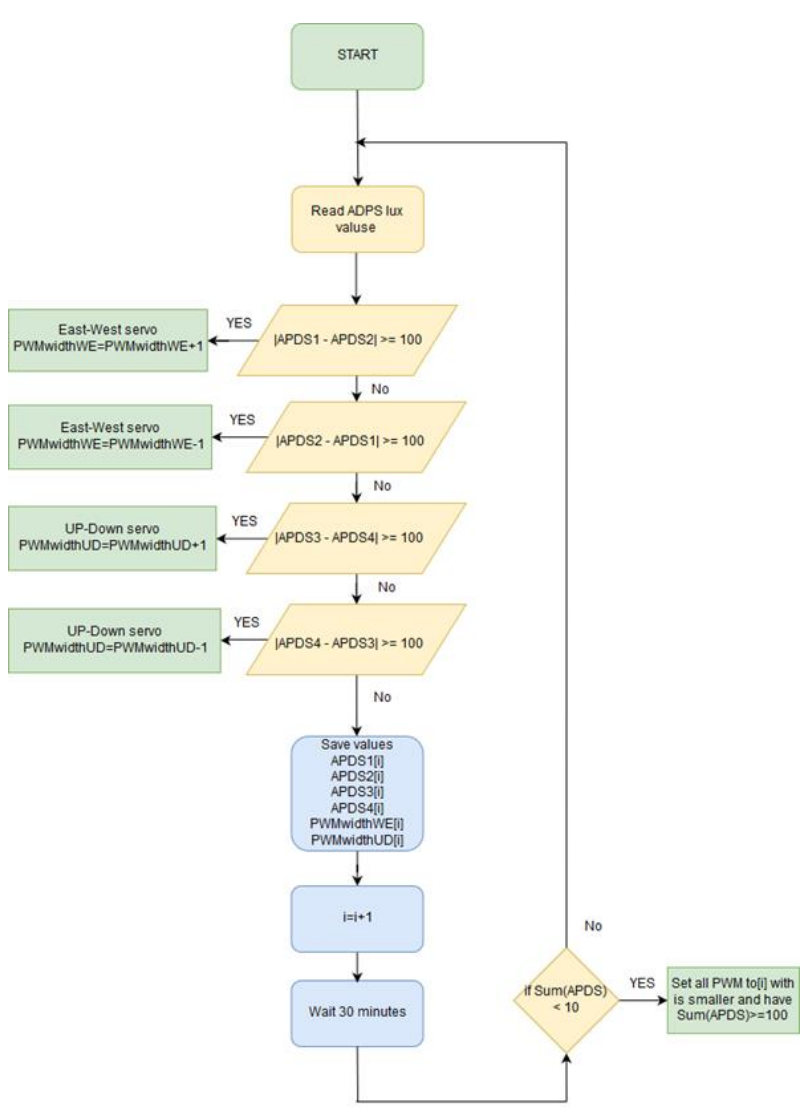

Fig. 3: The algorithm of the program

\section{Design of the target structure}

It is made of a cross-shaped base that can be trimmed with concrete blocks. The base is welded to the mast from an aluminium profile. The pole is finished with a hub with a rack. An electric motor placed next to it with a built-in gear is coupled with a gear. Thanks to this, an east-west rotation is possible. A T-shaped profile is welded to the top of the hub. The longer part of the frame is connected to the electric actuator and the shorter part to the PV panel mount. The use of bearings leads to enabling the change of the angle of photovoltaic panels, using one actuator. The electric motor and the linear electric motor have built-in encoders. The advantage of this design is its rigidity, ease of making, and a small number of moving parts. The weight of the entire structure without a solar panel is $38 \mathrm{~kg}$.

\section{Results and discussion}

The test platform has been set on the roof of a dormitory. The weather was windless and the sky was clear. In addition to the test platform, the same photovoltaic panel was mounted at an angle of $45^{\circ}$ and connected to a voltage meter. We carried out the tests throughout the day. The meters had a built-in memory in which measurements were recorded. The selected angle is optimal in the place of research.

Comparing (3) the sum of photovoltaic panel voltages $(1,2)$ in the Solar Tracker to a permanently mounted panel, we can see an increase in the generated voltage.

$$
\begin{aligned}
& \sum V 1=59,96 \\
& \sum V 2=70,91 \\
& \frac{\sum V 2-\sum V 1}{\sum V 1} * 100 \%=18,26 \%
\end{aligned}
$$

Thus, it can be seen that the use of Solar Tracker resulted in an 18\% (3) increase in the power generated by the solar panel relative to the panel, which was mounted at an angle of $45^{\circ}$. 


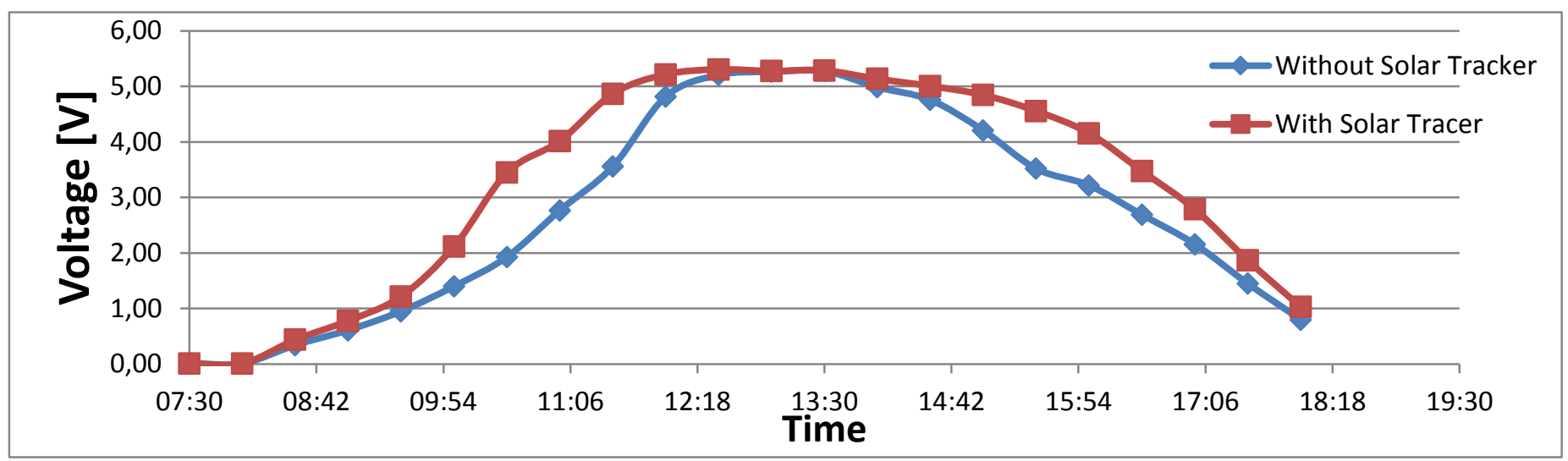

Fig. 4: Diagram of voltage measurement over time. Red - using Solar Tracker. Blue - without using Solar Tracker

\section{Conclusions}

The use of Solar Tracker allowed obtaining $18 \%$ more power generated by the photovoltaic panel. It should be emphasised that the power consumed by servomotors has not been measured, and the solar panel itself is cheap and inefficient. In the target structure for controlling electric motors, we would use DC Driver Pololu. In addition, the STM32 microcontroller has the ability to switch to low-energy mode, thanks to which all peripherals and sensors are switched off. Returning to normal operation would occur cyclically up to 30 minutes for a few seconds to correct the position of the Solar Tracker. Such a solution will allow maximising the acquisition of energy in relation to the expenditure using high-quality solar panels.

\section{Acknowledgement}

The work was partially financed by the Polish Ministry of Science and Higher Education program „Best of the best! 3.0." and from the means of the Faculty of Mechanical Engineering of the Bialystok University of Technology.

\section{References}

Sidharth Makhija, Aishwarya Khatwani, Mohd. Faisal Khan, Vrinda Goel, M. Mani (2017) International Conference on Inventive Systems and Control (ICISC) Roja Design \& implementation of an automated dual-axis solar tracker with data-logging 10.1109/ICISC.2017.8068708

Hamid Allamehzadeh (2016) IEEE Conference on Technologies for Sustainability (SusTech) Solar energy overview and maximizing power output of a solar array using sun trackers 10.1109/SusTech.2016.7897136.

Rocio Alba-Flores, Deon Lucien,Tricia Kirkland, Lindsay Snowden, Dallas Herrin (2018) SoutheastCon Design and Performance Analysis of three Photovoltaic Systems to Improve Solar Energy Collection 10.1109/SECON.2018.8478909.

J. Kern M. Russell (1978) in 13th IEEE Photovolt. Spec. Washington DC USA:Massachusetts Inst. of Tech. Lexington (USA) Combined photovoltaic and thermal hybrid collector systems Lincoln Lab pp. COO-4577-3-CONF780619-24.

S. Ozcelik H. Prakash R. Challoo (2011) Procedia Computer Science vol. 6 Two-axis solar tracker analysis and control for maximum power generation pp. 457-462.

G. Deb A.B. Roy (2012) International Journal of Computer and Electrical Engineering vol. 4 no. 1

Use of solar tracking system for extracting solar energy 